See discussions, stats, and author profiles for this publication at: https://www.researchgate.net/publication/323710249

\title{
Study of a Superconducting Magnetic Diverter for the ATHENA X-Ray Space Telescope
}

Article in IEEE Transactions on Applied Superconductivity · March 2018

DOI: 10.1109/TASC.2018.2811862

CITATION

1

6 authors, including:

8 Nicolò Riva

École Polytechnique Fédérale de Lausanne

5 PUBLICATIONS 2 CITATIONS

SEE PROFILE

Paolo Saracco

INFN - Istituto Nazionale di Fisica Nucleare

132 PUBLICATIONS 536 CITATIONS

SEE PROFILE

Some of the authors of this publication are also working on these related projects:

Project EPFL Hyperloop View project

Project Uncertain Quantification INFN View project
READS

64

INFN' Valerio Calvelli
INFN - Istituto Nazionale di Fisica Nucleare
39 PUBLICATIONS 795 CITATIONS
SEE PROFILE




\title{
Study of a Superconducting Magnetic Diverter for the ATHENA X-Ray Space Telescope
}

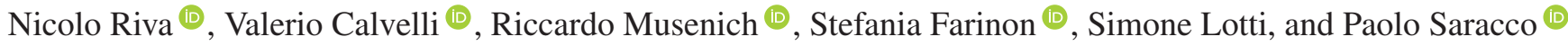

\begin{abstract}
The advanced telescope for high energy astrophysics (ATHENA) is an X-ray telescope of the European space agency which is planned to be launched in 2028. It will carry out observations in the $\mathrm{X}$-ray band, exploiting two focal plane detectors where $X$-ray photons will be focused by silicon pore optics. Previous $\mathrm{X}$-ray missions have shown a serious issue with soft protons and ions (below $150 \mathrm{keV} / \mathrm{n}$ ), which could enter the telescope mirror aperture and be concentrated toward the focal plane. These particles must be blocked or diverted to avoid excess background loading on the detectors. The proposed solution is to deflect protons away from the instruments field of view by means of magnets located between the optics and the focal plane. A 160000 amp-turns HTS superconducting toroidal magnet, composed by three or four round coils located between 0.6 and $1.2 \mathrm{~m}$ from the focal plane, can efficiently deflect most of the incoming particles. The rejection rate for protons till $120 \mathrm{keV}$ is better than $99 \%$, to be compared with about $80 \%$ of a previously proposed diverter based on permanent magnets. The magnetic field requirement at the detectors level (B $<1 \mathrm{mT}$ ) is widely satisfied, as well as the mass budget of $110 \mathrm{~kg}$. Challenging aspects related to the operation and reliability of the superconducting magnet will be discussed.
\end{abstract}

Index Terms-Advanced telescope for high energy astrophysics (ATHENA), ESA, genetic algorithms, HTS, magnetic diverter, $\mathrm{MgB}_{2}$, superconductors.

\section{INTRODUCTION}

$\mathbf{T}$ HE Advanced Telescope for High ENergy Astrophysics (ATHENA) is an X-ray telescope of the European Space Agency which is planned to be launched in 2028. It will be placed in a halo orbit around the Lagrangian point L2. The space-based observations in the X-ray band will be performed by the WFI (Wide Field Imager) and X-IFU (X-ray Integral Field Unit) detectors, where X-ray photons will be focused. Previous missions [6]-[10] have shown the necessity to block or deflect low energy particles concentrated by the mirrors, which may affect the sensitivity of the detectors or a induce radiation damage. So far, the proposed existing solution is the magnetic deflection using permanent magnets [2]. We studied a magnetic

Manuscript received September 18, 2017; accepted February 14, 2018. Date of publication March 8, 2018; date of current version April 5, 2018. This work was funded by INFN in the framework of the LAPUTA project. (Corresponding author: Nicolo Riva.)

N. Riva was with INFN and Università di Milano, Italy. He is now with Ecole Polytechnique Fédérale de Lausanne, Lausanne 1015, Switzerland (e-mail: nicolo.riva@epfl.ch).

V. Calvelli, R. Musenich, S. Farinon, and P. Saracco are with INFN, Genova 16146, Italy.

S. Lotti is with the INAF, Rome 00136, Italy.

Color versions of one or more of the figures in this paper are available online at http://ieeexplore.ieee.org.

Digital Object Identifier 10.1109/TASC.2018.2811862
TABLE I

GENERAL REQUIREMENTS

\begin{tabular}{lc}
\hline \hline Requirement & Value \\
\hline Overall Mass & $\leq 110 \mathrm{~kg}$ \\
Dipole Moment & $\sim 0 \mathrm{~A} \cdot \mathrm{m}^{2}$ \\
Photon Flux Block & $=0 \%$ \\
$|\boldsymbol{B}|$ on the WFI detector & $B_{\mathrm{W} \mathrm{F}}<10^{-3} \mathrm{~T}$ \\
$|\mathbf{B}|$ on the X-IFU detector & $B_{\mathrm{X}-\mathrm{IF} \mathrm{U}}<10^{-3} \mathrm{~T}$ \\
\hline \hline
\end{tabular}

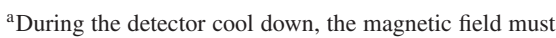
be $B_{\mathrm{X}-\mathrm{IF} \mathrm{U}}<10^{-4} \mathrm{~T}$.

diverter based on superconducting magnets, using optimization tools and sweep parameters analysis for the geometry and Monte Carlo particle tracking simulations for the particle fluxes.

The proposed solution is a toroidal magnet composed of 3 or 4 round coils, located between $0.6 \mathrm{~m}$ and $1.2 \mathrm{~m}$ from the focal plane.

\section{DESIGN REQUIREMENTS}

\section{A. Mission and Technical Requirements}

Table I shows the ATHENA mission requirements that directly impact the diverter design. The overall mass of the diverter and its ancillaries must be less than $110 \mathrm{~kg}$, and the incoming photon flux must not be blocked by the diverter materials. The residual magnetic dipole moment must be minimized to avoid any possible torque due to the interaction with the geomagnetic field.

Requirements on the residual magnetic flux density are instead due to the on board instrumentation. The sensitivity of the detectors, in fact, can be affected by the presence of a magnetic field at the focal plane level.

The diverter must operate in a thermal environment where the main source of heat is the radiation from the mirrors, which are kept at about $300 \mathrm{~K}$.

\section{B. Efficiency Requirements}

The requirements for the rejection efficiency are described in [1], [2]. Regarding the soft protons background, the largest uncertainty is due to the external fluxes impacting on the mirrors. In fact, the low energy environment in L2 is not well-known and it is highly dynamic, making hard to predict the impact on the mission.

However, the latest study on the particle background expected on X-IFU instrument is carried out in [6] with Monte Carlo 


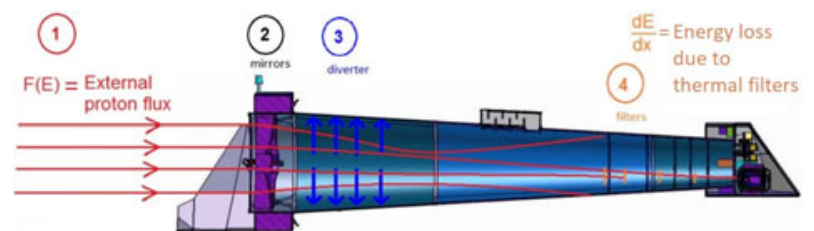

Fig. 1. Schematics of the path followed by the soft protons through the telescope. The filters shown (identified with the number 4) are thermal filters, which can absorb the lowest energy protons. In the work reported in [6], any magnetic field has been taken into account.

TABLE II

Max. Particle Energy to Be Deflected FROM FOCAL PLANE IN keV

\begin{tabular}{lcc}
\hline \hline Particles & WFI & X-IFU \\
\hline Protons & $75 \mathrm{keV}$ & $74 \mathrm{keV}$ \\
Electrons & $31 \mathrm{keV}$ & $21 \mathrm{keV}$ \\
\hline \hline
\end{tabular}

simulations, where the proton interactions with different parts of the telescope have been mapped without considering any magnetic field acting on their trajectories (see Fig. 1).

The result is the estimation of an energy threshold below which it is necessary to remove more than $96.5 \%$ of the incoming flux [6]. In Table II the thresholds are reported for protons and electrons.

\section{DESIGN SOLUTIONS}

In order to fulfill the mass requirement as well as to guarantee a high stability margin to the magnet, the choice of the superconductor is naturally led either to HTS or to $\mathrm{MgB}_{2}$ cables [3], [4]. We made a comparative analysis between Ti-clad $\mathrm{MgB}_{2}$ conductor and ReBCO tapes for each design, in order to identify which one most minimizes the mass of the winding and ancillary equipment at the same operative current density, here set at $80 \mathrm{~A} / \mathrm{mm}^{2}$. The operating temperature is set respectively to $20 \mathrm{~K}$ for the Ti- $\mathrm{MgB}_{2}$ conductor [4] and to around $70 \mathrm{~K}$ for the ReBCO, with a margin of about $50 \%$ on the critical current density @1 $\mathrm{T}$ in perpendicular field. The Ti-MgB 2 conductor density is about 2.1 times lower than ReBCO: $4000 \mathrm{~kg} / \mathrm{m}^{3} \mathrm{com}-$ pared to $8500 \mathrm{~kg} / \mathrm{m}^{3}$.

As regards ancillary equipment (mechanical structure, power supply, cryogenics, quench detector and protection system), the choice of the conductor mainly affects cryogenics as it is related to the operating temperature. The magnet cryogenics could be based on a passive cooling system as already used in PLANK NASA satellite [5]: cryogenic heat pipes connect the payload to radiators. Only four fluids are available to operate heat pipes below $70 \mathrm{~K}$ : hydrogen, deuterium, neon, and nitrogen. For Re$\mathrm{BCO}$, it is possible to use liquid nitrogen while for $\mathrm{MgB}_{2}$ the only suitable fluid is hydrogen. Respect to a ReBCO magnet operating at $70 \mathrm{~K}$, the $\mathrm{MgB}_{2}$ option requires larger radiators. Indeed, the power radiated toward the deep space is $1.2 \mathrm{~W} / \mathrm{m}^{2}$ at $70 \mathrm{~K}$ while it is only $8 \mathrm{~mW} / \mathrm{m}^{2}$ at $20 \mathrm{~K}$. The low radiating power at $20 \mathrm{~K}$ implies large panels or the use of a cryocooler. The consequent mass penalty and impact on the payload design steers the choice to a ReBCO magnet.

\section{Conceptual Designs And Optimization ToOls}

In order to consider all the requirements, we developed a series of optimization tools that allow us to minimize the mass, dimensions and total current (Ampere-turns). We decided to apply two different approaches to two slightly different designs. Basically, the design explored is a toroid with a low number of coils in order not to intercept the incoming photon flux and to limit the mass. On the other hand, the fringe field would be higher than in the usual toroidal configurations and the bending power lower. The optimization has been carried out taking care of those aspects, exploiting the parameters we considered critical for the diverter. For both designs, Monte Carlo simulations have been performed in order to estimate the entity of the particles rejection performance, a leading parameter of the design quality.

As the activity was devoted to a preliminary magnetic design, no hypothesis was done about the conductor dimension and, consequently, about the operating current. Therefore, some aspects of the magnet design, like quench protection, will be analyzed during further developments of the project.

\section{A. Genetic Algorithms Optimization}

One of the proposed designs is a toroidal diverter made of 3 racetrack coils, at which we applied an optimization process through the Evolutionary Algorithms (EAs). EAs are population-based meta-heuristic optimization algorithms that use biology-inspired mechanisms and survival of the fittest theory in order to refine a set of solution $\left\{x_{k}\right\}$ iteratively, that always converge to one solution. Genetic algorithms (GAs) are a subclass of (EAs) where the elements of the search space are binary strings or arrays of other elementary types [11].

For this work, we wrote a custom GA MatLab based code. First, it calculates the magnetic field obtained by the 3 coils toroidal diverter with COMSOL engine, then it tracks the particles in the calculated field (COMSOL). Through MatLab LiveLink the GA communicates with COMSOL on purpose to obtain solutions and parameters that the GA needs to calculate the Fitness Function (FF), then decides how to proceed in the evolution. The FF to minimize is defined as $\alpha_{d e t}=N_{d e t} / N_{i n c}$ : the fraction of particles intersecting the detectors $N_{\text {det }}$ ratio the incident particles $N_{i n c}$. The smaller $\alpha_{\text {det }}$ is, the greater is the efficiency of deflecting particles.

The design variables considered are geometrical and electrical: the coil dimensions, the distance from the field of view ( $\mathrm{z}$ coordinate) and number of Ampere-turns.

The constraints for the FF come from the design requirements, namely the total mass and distance of the diverter from the detector (which is related to the constraint Bpeak on the detectors). In Table III we report the design parameters and in Fig. 2 the GA concept design.

As results of this analysis, we found that with $\mathrm{MgB}_{2}$ the mass of the winding is $14 \mathrm{~kg}$, to which we have to add roughly $100 \mathrm{~kg}$ with the ancillaries, while with ReBCO the mass of the winding is $26 \mathrm{~kg}$ for the diverter and $50 \mathrm{~kg}$ with the ancillaries. 
TABLE III

THREE COIL TOROID SPECIFICATIONS

\begin{tabular}{|c|c|c|c|}
\hline Coil length & $350 \mathrm{~mm}$ & Coils aperture & $120^{\circ}$ \\
\hline Coil height & $450 \mathrm{~mm}$ & Ampere.turns & 160000 \\
\hline Cross-section & $600 \mathrm{~mm}^{2}$ & $\mathrm{~B}_{\max }$ over conductor & $1.07 \mathrm{~T}$ \\
\hline$x^{\mathrm{b}}$ & $30 \mathrm{~mm}$ & Energy stored & $4300 \mathrm{~J}$ \\
\hline$y^{\mathrm{b}}$ & $200 \mathrm{~mm}$ & Dipole Moment & $0 \mathrm{Am}^{2}$ \\
\hline \multirow[t]{2}{*}{$z^{\mathrm{b}}$} & $1205 \mathrm{~mm}$ & $\mathrm{SC}$ mass $\left(\mathrm{MgB}_{2}\right)$ & $14 \mathrm{~kg}$ \\
\hline & & $\mathrm{SC}$ mass (REBCO) & $26 \mathrm{~kg}$ \\
\hline
\end{tabular}

${ }^{a}$ Inner and outer radius of the toroidal magnet.

${ }^{b}$ Coordinates of the diverter center of mass respect to the axis of the telescope and the detector's plane.

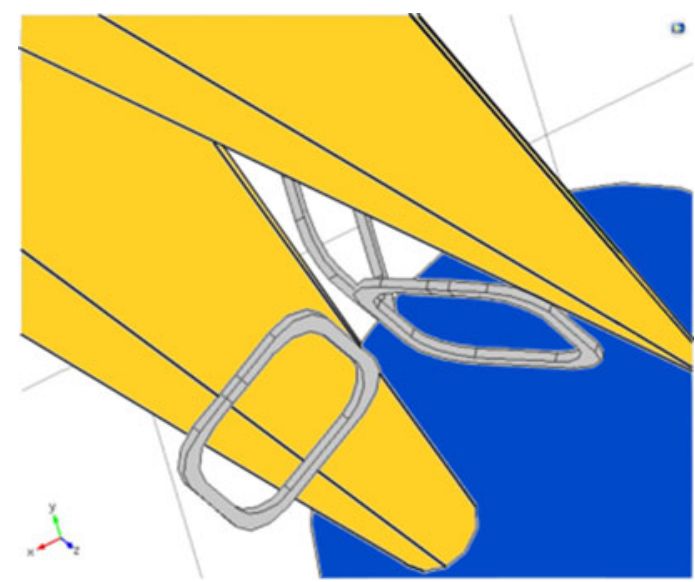

Fig. 2. First concept design of the diverter with the photons flux incident on the detectors. The blue surface is the plane where detectors lie, meanwhile the yellow cone are the particle fluxes.

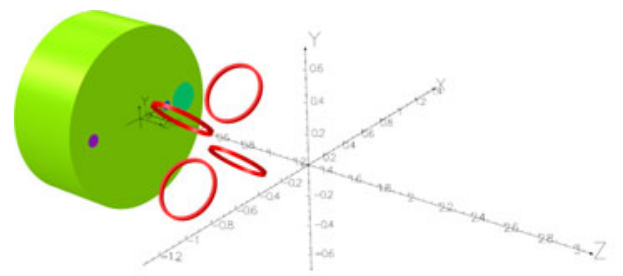

Fig. 3. Second concept design of the diverter.

\section{B. Sweep Parameters Analysis}

The second diverter proposed is a 4 coils toroid (see Fig. 3) which design has been selected through a parametric model of 10 degrees of freedom. They are respectively the Cartesian coordinate $x, y, z$ needed to find the best position of the diverter center of mass respect to the axis of the telescope and the detectors plane; the geometric dimensions of each coil (inner and outer radius $r_{i}, r_{e}$ of the toroid, width $w$ of the coil); two Euler angles $\hat{x}, \hat{y}$ related to the $x, y$ axis, to identify the best rotational position of the center of mass; and two angles $\hat{c}_{1}, \hat{c}_{2}$ that identify the azimuthal position of each coils respect to the detectors position, in order to avoid any block of the photons flux.

The model has been prepared using OPERA by Cobham, swapping the parameters to find the best configuration using an in-house code. As results, the mass of the diverter with $\mathrm{MgB}_{2}$ is $9.5 \mathrm{~kg}$, while for YBCO is $20.2 \mathrm{~kg}$. Also for this design, the
TABLE IV

FOUR COIL TOROID SPECIFICATIONS

\begin{tabular}{lccc}
\hline \hline DoFs & \multicolumn{3}{c}{ Specifications } \\
\hline$r_{i}$ & $150 \mathrm{~mm}$ & Ampere-turns & 160000 \\
$r_{e}$ & $330 \mathrm{~mm}$ & B $_{\mathrm{m} \text { ax }}$ over conductor & $0.74 \mathrm{~T}$ \\
$w$ & $25 \mathrm{~mm}$ & Energy stored & $2100 \mathrm{~J}$ \\
$x$ & $80 \mathrm{~mm}$ & Dipole Moment & $0 \mathrm{Am}^{2}$ \\
$y$ & $80 \mathrm{~mm}$ & SC mass (MgB $)$ & $9.5 \mathrm{~kg}$ \\
$z$ & $600 \mathrm{~mm}$ & SC mass (YBCO) & $20.2 \mathrm{~kg}$ \\
$\hat{x}$ & $0^{\circ}$ & & \\
$\hat{y}$ & $0^{\circ}$ & & \\
$\hat{c}_{1}$ & $45^{\circ}$ & \\
$\hat{c}_{2}$ & $45^{\circ}$ & \\
\hline \hline
\end{tabular}
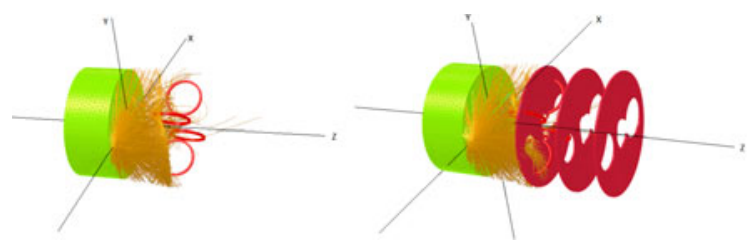

Opera

Fig. 4. Reverse tracking simulations without filters on the left, with filters on the right.

mass for the ancillary systems are evaluated as about $100 \mathrm{~kg}$ for the $\mathrm{MgB}_{2}$ and $50 \mathrm{~kg}$ for the ReBCO configuration. Both analyses point out that a good solution for the diverter can be the ReBCO; in fact, low mass and a cryocooler free system is more suitable for this application. The design specifications are reported in Table IV.

\section{Monte Carlo Particle Tracking Simulations}

Charged particles rejection performances of both designs have been estimated through Monte Carlo simulations. We used respectively COMSOL for the 3-coils and OPERA for the 4-coils designs. For both softwares, we wrote a dedicated code to perform the simulations in order to consider the transportation properties and the interactions with the magnetic field for protons. Being those simulations used to estimate and not to validate the designs performances, other particles and nuclear interactions have not been considered, as well as secondary particles production. For both designs we used at first a Monte Carlo direct particle tracking: the simulated proton beam hitting the detectors from the telescope mirrors is modeled as a uniform angular spread with the same rms width in the sagittal and meridional planes. Simulations have been carried out with different $r m s$ and energies, then tracked under the effect of the magnetic field produced by the diverter. For these simulations, no thermal filter (Figs. 1, 4) has been modeled. In order to have a better statistics and a more reliable validation including the effects on the particles produced by the thermal filters of the telescope, for the 4-coils design we used also the reverse particle tracking: particles have been released from the detectors with a certain solid angle, the magnetic field was inverted and the particles were tracked in order to see which ones were effectively coming from the mirror aperture of the telescope. The results are presented afterward. 
TABLE V

\begin{tabular}{lcccc}
\hline \hline $\mathrm{rms}$ & $20 \mathrm{keV}$ & $75 \mathrm{keV}$ & $120 \mathrm{keV}$ & $500 \mathrm{keV}$ \\
\hline 0.5 & $<1 \cdot 10^{-5}$ & $<1 \cdot 10^{-5}$ & $<1 \cdot 10^{5}$ & $<1 \cdot 10^{5}$ \\
1 & $<1 \cdot 10^{-5}$ & $3.53 \cdot 10^{-5}$ & $<1 \cdot 10^{5}$ & 0.001 \\
2 & $<1 \cdot 10^{-5}$ & $3.22 \cdot 10^{-4}$ & $2.01 \cdot 10^{-4}$ & 0.0028 \\
4 & $<1 \cdot 10^{-5}$ & $1.56 \cdot 10^{-4}$ & $2.23 \cdot 10^{-4}$ & 0.0035 \\
\hline \hline
\end{tabular}

WFI - Protons Reduction without Thermal Plates (\%)

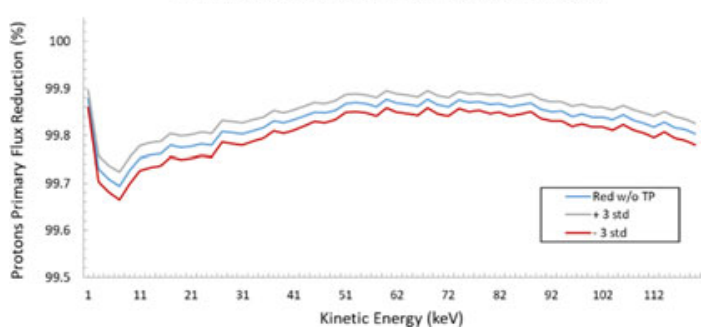

Fig. 5. Protons reduction in percentage for the WFI detector without thermal plates as a function of the kinetic energy of the entering spectra. The grey and red lines are respectively the statistical uncertainties at $\pm 3 \sigma$.

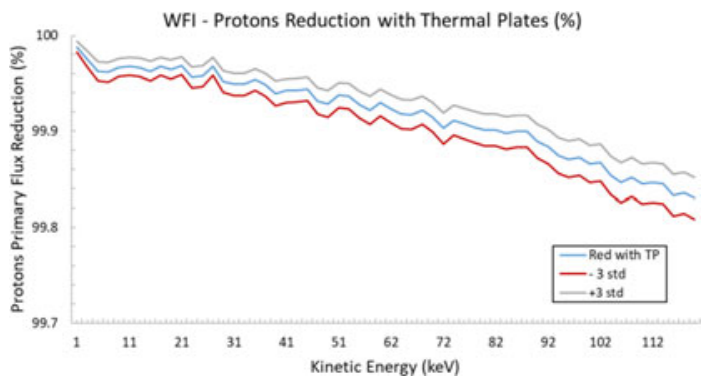

Fig. 6. Protons reduction in percentage for the WFI detector with thermal plates as a function of the kinetic energy of the entering spectra. The grey and red lines are respectively the statistical uncertainties at $\pm 3 \sigma$.

\section{A. 3-Coils Diverter}

In order to be consistent with the efficiency evaluations reported in [2], the simulations were carried out with $3 \cdot 10^{5}$ particles directed to the focal plane of the detectors with energies from $20 \mathrm{keV}$ to $500 \mathrm{keV}$ and rms from 0.5 to 1 . In the simulations, no thermal filters were considered. In Table V, it is reported the efficiency of the diverter obtained with GA optimization for the detector WFI, which present higher value of $\alpha_{\text {det }}$ because of his size. From $\alpha$ we calculate the efficiency rejection as $\eta=100 \cdot(1-\alpha)$. In some cases, the efficiency at lower energy seems less than at higher energy, e.g., at $75 \mathrm{keV}$ for $r m s=2$ the efficiency is lower than at $150 \mathrm{keV}$. Even if the number of particles is not high enough to get a good statistic we may suppose that this is due to particles that swirl around the diverter because of the intensity of the field. Those particles may be deflected radially inward and directed to the detectors instead of being deflected radially outward. Due to computational limits, it was not possible to achieve a better statistics.

\section{B. 4-Coils Diverter}

We simulated in more details the proton spectra entering the WFI and X-IFU detectors in the energy region of
0-120 keV, considering also the effects of the thermal filters (see Fig. 4).

Simulations were carried out with $4.1 \cdot 10^{7}$ protons each one, in order to achieve a significant statistic. Results for the WFI, the worst one in terms of reduction, are shown in Fig. 5, 6. The results obtained without thermal plates show that a small fraction of protons (especially at the energy of few $\mathrm{keV}$ ) follows the external lines of the magnetic field, swirling around the diverter and hitting the detectors. With thermal plates, this effect is suppressed because those protons are absorbed by the materials.

\section{CONCLUSION}

After an initial trade-off analysis, a 160000 amp-turns HTS superconducting toroidal magnet, composed of 3 or 4 round coils has been chosen and a preliminary conceptual design. The magnet can be wound with a high-temperature superconductor $\left(\mathrm{MgB}_{2}\right.$ or preferably ReBCO). Differently, from the permanent magnet, positioned close to the mirrors, the proposed superconducting diverter is located between $0.6 \mathrm{~m}$ and $1.4 \mathrm{~m}$ from the focal plane.

Challenging aspects are related to the operation and reliability of the superconducting magnet, namely cryogenics, stability and quench protection. Nevertheless, these solutions are, at this level of study, really competitive with the other proposed for ATHENA in terms of both efficiency and mass. The rejection rate for protons at $120 \mathrm{keV}$ is better than $99.8 \%$, to be compared with the about $80 \%$ of the diverter based on permanent magnets. The requirements on the magnetic field on the detectors and the mass are widely satisfied.

This feasibility study has revealed that a superconducting diverter could be an efficient and interesting solution for the ATHENA X-rays telescope.

\section{REFERENCES}

[1] ATHENA L2 Payload Definition Document, 2015, European Space Agency, Paris, France, CFD

[2] R. Willingale et al., ATHENA Supporting Paper, 2014.

[3] R. Musenich, V Calvelli, S Farinon, W. J Burger, and R. Battiston, "Space radiation superconducting shields," J. Phys. Conf. Ser, vol. 507, 2014, Art. no. 032033.

[4] R. Musenich et al., "Ti-MgB2 conductor for superconducting space magnets," IEEE Trans. Appl. Supercond., vol. 26, no. 4, Jun. 2016, Art. no. 6200204. doi: 10.1109/TASC.2015.2509171.

[5] L. A. Wadel et al., "Hydrogen sorption cryocoolers for the Planck mission," 1999. [Online]. Available: http://hdl.handle.net/2014/18308

[6] S. Loti et al., The particle background of the X-IFUinstrument. 2017. doi: 10.1007/s10686-017-9538-1.

[7] S. Molendi presentation at XMM radiation environment workshop. 2000. [Online.] Available: http://xmm2.esac.esa.int/docs/documents/Appendix /molendi_0001a.tif.pdf.gz

[8] A. De Luca and S. Molendi, "The 2-8 keV cosmic X-ray background spectrum as observed with XMM-Newton," $A \& A$, vol. 419, pp. 837-848, 2004, doi: 10.1051/0004-6361:20034421.

[9] K. D. Kuntz and S. L. Snowden, "The X-ray-emitting components toward $1=111^{\circ}$ : The local hot bubble and beyond," Astrophys. J., vol. 674, pp. 209-219, 2008.

[10] K.D. Kuntz presentation at IACHEC meeting. 2014. [Online]. Available: http://web.mit.edu/iachec/meetings/2014/Presentations/Kuntz.pdf

[11] R. L. Haupt and D. H. Werner, Genetic Algorithms in Electromagnetics. Hoboken, NJ, USA: Wiley, 2007.

[12] A. Leccardi and S. Molendi, "Radial temperature profiles for a large sample of galaxy clusters observed with XMM-Newton," A\&A, vol. 486, pp. 359-373, 2008 . 